\title{
A QUALIDADE DE VIDA DOS PROFESSORES DE EDUCAÇÃO FÍSICA ESCOLAR DA REDE PÚBLICA E PRIVADA DE CATOLÉ DO ROCHA-PB
}

\author{
Alana Simões Bezerra \\ Matheus Vieira da Silva ${ }^{2}$
}

\section{Resumo}

O estilo de vida dos professores de Educação Física, as longas jornadas de trabalho e os baixos salários, faz com que esses profissionais tenham fixação pelo trabalho impactando na saúde e na qualidade de vida. A pesquisa teve como objetivo avaliar a qualidade de vida dos professores de Educação Física escolar da rede pública e privada de Catolé do Rocha-PB. Trata-se de uma pesquisa direta, de campo e de abordagem quantitativa. Participaram da pesquisa professores de Educação Física da rede pública e privada da cidade de Catolé do Rocha-PB. Como instrumento da pesquisa, utilizou-se o questionário The Medical Outcomes Study 36 - Short Form Health Survey (SF-36). Observa-se que a maioria dos dados adquiridos em cada domínio não alcança o valor de 100 que é dado como melhor saúde. Porém tem-se a capacidade funcional (93), a limitação aspecto físico (80) e a dor $(63,2)$ como bons valores quanto a saúde do professor da rede privada. Já na rede pública apenas os domínios da capacidade funcional (65) e da dor (63,2). Já os domínios da dor, do estado geral de saúde, vitalidade, aspectos sociais, limitação dos aspectos emocionais e a saúde mental tiveram um equilíbrio entre os professores das instituições públicas e privadas. Assim, esta pesquisa constatou que a amostra estudada tem uma percepção mediana quanto a sua qualidade de vida.

Palavras-chave: Qualidade de vida. Professores. Educação Física.

\begin{abstract}
The lifestyle of Physical Education teachers, long working hours and low wages, makes these professionals have a fixation for work impacting on health and quality of life. The objective of the research was to evaluate the quality of life of the teachers of Physical Education in the public and private schools of Catolé do Rocha-PB. This is a direct field research and a quantitative approach. Participated in the research Physical Education teachers of the public and private network of the city of Catolé do Rocha-PB. As a research tool, the questionnaire The Medical Outcomes Study 36 - Short Form Health Survey (SF-36) was used. It is observed that most of the data acquired in each domain does not reach the value of 100 that is given as better health. However, functional capacity (93), physical aspect limitation (80), and pain (63.2) are considered as good values for the private teacher's health. Already in the public network only the domains of functional capacity (65) and pain (63.2). On the other hand, the domains of pain, general health, vitality, social aspects, limitation of emotional aspects and mental health had a balance between teachers of public and private institutions. Thus, this research found that the sample studied has a median perception of their quality of life.
\end{abstract}

Keywords: Quality of life. Teachers. Physical education.

1. Mestre em Ciências das Religiões e Professora Universitária na Faculdades Integradas de Patos - FIP (orientadora). 2. Graduado em Bacharelado em Educação Física

Autor para correspondência: Alana Simões Bezerra. Rua: Dr. José Genuino, 1166. Liberdade. Patos-PB. 


\section{INTRODUÇÃO}

Qualidade de vida é a percepção que o indivíduo tem de sua posição na vida no contexto cultural e no sistema de valores que ele vive em relação aos seus objetivos, expectativas, padrões e preocupações $^{1}$. A busca por uma boa qualidade de vida nos últimos anos está crescendo, pois a sociedade percebe sua relação com muitas características, como o bem estar, com isso está acontecendo um aumento pela procura das atividades físicas ${ }^{2}$.

Apesar dos profissionais de Educação Física serem responsáveis por promover qualidade de vida, o excesso de trabalho pode acarretar alguns problemas que afetam sua saúde, como angústia, fobias e crises de pânico ${ }^{3}$. Existe um conjunto de fatores que pode afetar na qualidade de vida, os baixos salários, os conflitos com os alunos e familiares pode gerar um conjunto de mal-estar, algumas doenças relacionadas ao trabalho como hipertensão arterial, doenças coronarianas, distúrbios mentais, estresse e câncer influenciam diretamente na qualidade de vida dos docentes ${ }^{4}$.

A qualidade de vida também está relacionada, ao local de trabalho, ao trabalhar em salas de aula lotadas, e locais com infraestrutura ruim e a jornada de trabalho excessivamente alta $^{5}$. Assim, todas essas situações configuram fatores psicossociais relacionados ao trabalho que pode resultar em sobrecargas de trabalho físicas e mentais e em consequências para a qualidade de vida dos professores ${ }^{6}$.

A experiência empírica com a qualidade de vida dos professores de Educação Física é relevante para os estudos atuais, tendo em vista que as produções científicas que abordam tal assunto são inúmeras, porém nenhum trabalho nessa proporção havia sido realizado entre os professores da cidade de Catolé do Rocha-PB. Essa pesquisa torna-se importante com essa população, pois Catolé do Rocha é uma das cidades do Sertão paraibano que mais cresce no índice de educação, porém não se sabe se este crescimento tem interferido na qualidade de vida dos professores. A pesquisa tem como objetivo avaliar a qualidade de vida dos professores de Educação Física da rede pública e privada de Catolé do RochaPB.

\section{MÉTODOS}

Trata-se de uma pesquisa de campo, pois se observa os fatos e fenômenos exatamente como acontece no real, com base numa fundamentação teórica consistente, tendo como objetivo 
compreender e explicar o problema da pesquisa $^{7}$. Para o desenvolvimento e aplicação desse estudo foi utilizado o método quantitativo, que requer o uso de estatística. É também um estudo comparativo.

A população da amostra se constitui por todos os professores de Educação Física da cidade de Catolé do Rocha-PB, sendo um total de 10 profissionais, destes 05 são professores da rede pública e 05 da rede privada. Dos 10 docentes que contribuíram para a amostra deste estudo apenas 1 era do sexo feminino e 9 do sexo masculino, com idade entre 35 e 55 anos.

Como instrumento da pesquisa, foi utilizado o The Medical Out Comes Study 36 - Item Short Form Health Survey (SF-36), é um questionário válido, confiável e disponível para utilização no Brasil, foi traduzido em 1997 por Ciconelli et al. ${ }^{8}$, e foi desenvolvido por Ware e Sherbourne ${ }^{9}$.

$$
\text { É um questionário }
$$

multidimensional formado por 36 itens, divididos em 8 domínios, são eles: capacidade funcional (10 itens), aspectos físicos (4 itens), dor (2 itens), estado geral de saúde (5 itens), vitalidade (4 itens), aspectos sociais (2 itens), aspectos emocionais (3 itens) e saúde mental (5 itens) e ainda, uma questão de avaliação comparativa entre as condições de saúde atual e a de um ano atrás. Este questionário avalia aspectos negativos relacionados à saúde (doença ou enfermidade) com aspectos positivos (bem-estar) ${ }^{10}$.

Antes de iniciar a pesquisa os pesquisadores responsáveis realizaram uma orientação para preenchimento do questionário. Os Professores de Educação Física receberam os questionários nas instituições de ensino que trabalham. Enquanto respondiam ao questionário, os aplicadores estavam disponíveis para qualquer tipo de esclarecimento. Após o recolhimento dos questionários, os dados foram analisados em planilha de Excel (2007) e foi calculada a média dos dados obtidos. Desta forma, os dados foram avaliados mediante análise quantitativa, verificando a qualidade de vida dos profissionais de Educação física de Escolas públicas e privadas do município de Catolé do Rocha-PB.

Foi realizado o cálculo do escore do SF-36. Nesta fase transformou-se os valores das questões do questionário em notas de 8 domínios que variam de 0 a 100 , onde $0=$ pior e $100=$ melhor para cada domínio, sendo analisado cada domínio separado, essa fase é chamada de raw scale, por que o valor final não apresenta nenhuma unidade de medida. Não existe um único valor que resuma 
toda a avaliação, traduzindo-se num estado geral de saúde melhor ou pior, justamente um sistema de escores, evite-se o erro de não se identificar os verdadeiros problemas de saúde ou mesmo subestimá-los ${ }^{8}$. Utilizou-se a estatística descritiva com determinação dos escores. A tabela 1 descreve a classificação dos escores de qualidade de vida dos professores.

$\mathrm{O}$ projeto foi submetido e aprovado pelo Comitê de Ética das Faculdades Integradas de Patos (CAEE: 58758016.5.00000.5181). Também foi elaborado um Termo de Consentimento Livre e Esclarecido para ser assinado pelos professores.

Tabela 1. Escala de pontuação para classificação 0 a 100 (SF-36)

\begin{tabular}{ll}
\hline PONTOS & CLASSIFICAÇÃO \\
$\mathbf{0 - 1 0 0}$ & \\
\hline $\mathbf{1 0 0}$ & Excelente \\
$\mathbf{8 4}$ & Muito Bom \\
$\mathbf{6 1}$ & Bom \\
$\mathbf{2 5}$ & Regular \\
$\mathbf{0}$ & Ruim \\
\hline
\end{tabular}

\section{RESULTADOS E DISCUSSÃO}

Os resultados da pesquisa referente a avaliação da qualidade de vida dos professores estão na tabela 2 , que apresenta os escores de cada domínio do questionário SF-36.

Diante dos resultados obtidos, pode-se observar que a maioria dos dados adquiridos em cada domínio não alcança o valor de 100 que é dado como melhor qualidade de vida. Porém tem-se a capacidade funcional (93), a limitação aspecto físico (80) e a dor $(63,2)$ como bons valores quanto a saúde do professor da rede privada, ambos podem ser classificados como excelente e bom estado de saúde. Já na rede pública apenas os domínios da capacidade funcional $(65)$ e da dor $(63,2)$ tiveram um valor próximo de 100, sendo classificados como bom estado de saúde. Alguns domínios como aspectos sociais, limitação aspectos emocionais e saúde mental tiveram escores abaixo de 50, mais próximos de zero, percebendo assim um regular estado de saúde nesses domínios tanto dos professores das escolas da rede pública quanto os da rede privada (Tabela 2).

No gráfico 1, encontra-se o comparativo dos resultados obtidos, no que se relaciona à qualidade de vida dos professores da rede pública e privada das escolas da cidade de Catolé do Rocha/PB. Percebe-se que os domínios da dor, do estado geral de saúde, vitalidade, aspectos sociais, limitação dos aspectos emocionais e a saúde mental tiveram um equilíbrio entre os professores das instituições públicas e privadas, não obtendo uma diferença significativa. 
De acordo com os escores, os professores das escolas da rede privada possuem escores maiores nos domínios da capacidade funcional e limitação do aspecto físico, em relação aos professores das escolas públicas.

Tabela 2. Valores dos escores de qualidade de vida dos professores de Educação Física da rede pública e privada do município de Catolé do Rocha/PB de acordo com os domínios do SF-36.

\begin{tabular}{lcc}
\hline \multicolumn{1}{c}{ Domínios } & $\begin{array}{c}\text { Rede } \\
\text { Privada }\end{array}$ & $\begin{array}{c}\text { Rede } \\
\text { Pública }\end{array}$ \\
\hline $\begin{array}{l}\text { Capacidade } \\
\text { Funcional }\end{array}$ & 93 & 65 \\
$\begin{array}{l}\text { Limitação } \\
\text { Aspecto Físico }\end{array}$ & 80 & 55 \\
$\begin{array}{l}\text { Dor } \\
\text { Estado Geral } \\
\text { de Saúde }\end{array}$ & 63,2 & 63,2 \\
$\begin{array}{l}\text { Vitalidade } \\
\begin{array}{l}\text { Aspectos } \\
\text { Sociais }\end{array}\end{array}$ & 49 & 49 \\
$\begin{array}{l}\text { Limitação } \\
\text { Aspectos } \\
\text { emocionais }\end{array}$ & 32 & 42 \\
Saúde Mental & 35,2 & 25 \\
\hline
\end{tabular}

De acordo com os escores, os professores das escolas da rede privada possuem escores maiores nos domínios da capacidade funcional e limitação do aspecto físico, em relação aos professores das escolas públicas.

No estudo de Rocha e Fernandes $^{11}$ realizado em escolas municipais os resultados da capacidade funcional obteve os maiores resultados $(65,71)$, o que leva a interpretação que o grupo analisado possui boa capacidade funcional.

Os domínios de limitação dos aspectos emocionais dos professores da rede pública $(22,5)$ e privada (30), e também, o domínio aspectos sociais dos professores das escolas públicas (25) e privada (32) apresentaram valores regulares.

Gráfico 1. Distribuição dos professores da rede pública e privada da cidade de Catolé do Rocha-PB segundo a qualidade de vida.

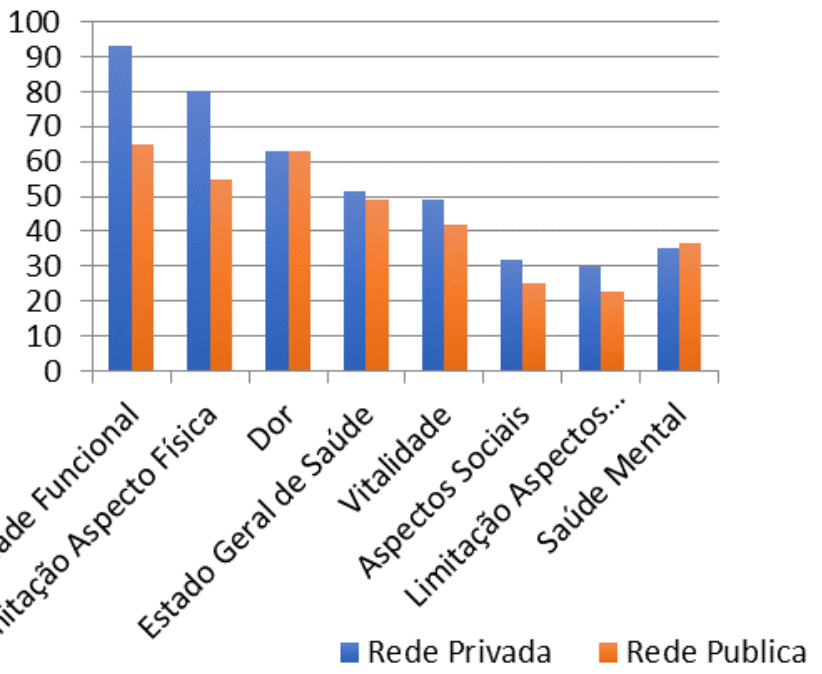

Em concordância com o nosso estudo, Silveira, Reis, Santos, Borges ${ }^{12}$ apresentaram em sua pesquisa que os domínios da limitação por aspectos emocionais e aspectos sociais se referem aos relacionamentos no ambiente familiar, nos lugares de convívio coletivo e no local de trabalho. Em seus estudos, esses domínios apresentaram valores regulares, indicando que os professores de 
educação física praticamente anulam seu convívio social e, por isso, podem estar mais expostos a alterações em sua saúde mental.

Em contrapartida com os resultados no nosso estudo, no que se refere ao domínio emocional, no qual obtivemos uma média regular, os estudos Rocha, Felli ${ }^{13}$ afirmam que todos os trabalhos geram vários fatores influenciam muito nos processos de saúde de todos os professores durante o trabalho e principalmente na sua qualidade de vida, principalmente a limitação aspectos emocionais que foi muito abaixo, pois o aspecto emocional tem sido o principal obstáculo dos professores dentro das salas de aulas.

Em pesquisa realizada por Martins $^{14}$, o resultado encontrado para cada domínio como, por exemplo, capacidade funcional está relacionada à aptidão física, isso leva em consideração a capacidade de realização de atividades na vida diária do professor, que sempre tem que superar e resistir os estresses e os desafios da vida.

O domínio aspecto social envolve vários fatores como a interação familiar, com amigos e a comunidade em geral, pois na presente pesquisa esse domínio teve a pontuação abaixo da média tanto nas escolas privadas quanto nas escolas públicas. Para Gonçalves, Penteado, Silvério ${ }^{15}$ a relação professor, alunos e direção da escola, pode ser determinante e importante para a saúde do professor sendo sugerido que estudos para essa população sejam mais amplos e interdisciplinares. Em outro estudo os autores afirmam que o ambiente escolar, as condições do trabalho e a organização do trabalho repercutem no processo saúde-doença. Afetando aspectos relacionados a tensão emocional provocados pelo excesso de trabalho, a privação do lazer e os distúrbios musculoesqueléticos ${ }^{16}$.

Cabe ressaltar que características como o trabalho intenso e repetitivo, a baixa remuneração, a falta de continuidade de perspectivas de carreira, os conflitos e as hostilidades sofridas, dentro outros, tem condicionado distúrbios psicológicos em docentes, podendo chegar a $41,50 \%$ desta classe trabalhadora ${ }^{12}$.

\section{CONSIDERAÇÕES FINAIS}

Observou-se neste estudo que as médias das respostas ao questionário The Medical Out Comes Study 36 Item Short Form Health Survey - SF-36 tiveram um equilíbrio quanto alguns domínios como, o da dor, do estado de saúde geral, vitalidade, aspectos sociais, limitação dos aspectos emocionais e a saúde mental. Apenas os domínios da 
capacidade funcional e da limitação física apresentou diferença entre os escores de professores da rede pública e privada. Esta pesquisa constatou que, entre professores desta amostra a Bezerra, A. S., e da Silva, M. V., 2018 qualidade de vida.

Porém, tendo em vista que a amostra foi pequena, pois a cidade de Catolé do Rocha-PB tem apenas 10 professores de educação física entre a rede pública e privada, novos estudos, mais amplos e representativos devem ser realizados em outros municípios do estado da Paraíba, para que se possa fazer uma análise geral da qualidade de vida dos professores do ensino básico de municípios da Paraíba/PB.

\section{REFERÊNCIAS}

1. ORGANIZAÇÃO MUNDIAL DE SAÚDE. Promoção de la salud: glossário. Genebra: OMS; 1998.

2. SEIDL, Eliane Maria Fleury; ZANNON, Célia Maria Lana da Costa. Qualidade de Vida e saúde: aspectos conceituais e metodológicos. Caderno Saúde Pública, v. 20, n. 2, p. 580-588, 2004.

3. WONG, Wong S.; CHANG, Simon T. M.; FUNG, Vivian B. K.; FIELDING, Richard. The differential mediating effects of pain and depression on the physical and mental dimension of quality of life in Hong Kong Chinese adults. Health Qual Life Outcomes, v.8, p. 1-6, 2010.

4. AMORIM, Rodrigo Oliveira; NASCIMENTO, Bianca Bueno; KRUG, Marília Rosso; NASCIMENTO, Karine Bueno. Qualidade de vida e nível de atividade de professores da rede municipal de ensino do município de Cruz Alta, RS. EFdesportes.com, Revista digital, v. 152, 2014.

5. ALMEIDA, Daurilene Rodrigues Sodré; LIMA, David Couto Araújo. Nível de atividade física da rede pública de ensino de Taguatinga-DF. 2014. 16f. Trabalho de Conclusão de Curso (monografia). Licenciatura em Educação Física, Universidade Católica de Brasília; Brasília, 2014.

6. FOLLE, Alexandra; FARIAS, Gustavo. Nível de qualidade de vida a atividade física de professores de escolas públicas estaduais da cidade de Palhoça (SC). Revista Mackenzie de Educação Física e Esporte, v. 11, n. 1, p. 11-21, 2012

7. MINAYO, Maria Cecília de Sousa, GOMES, Suely Ferreira Deslandes. Pesquisa social. 30. ed. Petrópolis: Editora Vozes, 2011.

8. CICONELLI, Rozana Maria. Tradução para o português e validação do questionário genérico de avaliação de qualidade de vida. 1997. 148f. Tese (doutorado). Doutorado em Medicina, Universidade Federal de São Paulo (EPM), São Paulo, 1997.

9. WARE, John E.; SHERBONE, Cathy Donald. The Mos 36 - Item Short Form Health Survey (SF-36): I. Conceptual Frameworl and Item selection. Medical Care, v. 30, n. 6, p. 473-483, 1992.

10. ABREU, Isabella Schroeder. Qualidade de vida relacionada à saúde de pacientes em hemodiálise no município de Guarapuava-PR. 2005. 83f. dissertação (mestrado). Mestrado em enfermagem, Universidade de São Paulo, Ribeirão Preto, 2005.

11. ROCHA, Vera Maria; FERNANDES, Marcos Henrique. Qualidade de vida de professores do ensino fundamental: uma perspectiva para a formação da saúde do trabalhador. J Bras Psiquiatria, v. 57, n. 1, p. 23-27, 2008. 
12. SILVEIRA, Rodrigo Eurípede; REIS, Nayara Araújo; SANTOS, Alvaro da Silva; BORGES, Maritza Rodrigues. Qualidade de vida de docentes do ensino fundamental de um município brasileiro. Revista de enfermagem referência, n. 4, p. 115123, 2011.

13. ROCHA, Sandra de Sousa Lima; FELLI, Vanda Elisa Andres. Qualidade de vida no trabalho docente em enfermagem. Rev Latino-Am Enfermagem, n. 12, p. 28-35, 2004.

14. MARTINS, Marcelle de Oliveira. Estudo dos fatores determinantes da prática de atividades físicas de professores universitários. 2005. 183f. Dissertação (mestrado). Mestrado em Educação Física, Universidade Federal de Santa Catarina (UFSC), Santa Catarina, 2000.

15. GONÇALVES, C. G. O.; PENTEADO, R. Z.; SILVÉRIO, K. C. A. Fonoaudiologia e saúde do trabalhador: a questão da saúde vocal do professor. Saúde em Revista, v. 7, n. 15, p. 45-51, 2005.

16. MEIRA, Thiago Raphael Martins; CARDOSO, Jefferson Paixão; VILELA, Alba Benemérita Alves, AMORIM, Camila Rego; ROCHA, Saulo Vasconcelos; ANDRADE, Ariane Nepomuceno; FREIRE, Daíla Santos. Percepção de professores sobre trabalho docente e repercussões sobre sua saúde. Rev. Bras. Promoç. Saúde, v.27, n. 2, p. 276-282, 2014. 
Bezerra, A. S., e da Silva, M. V., 2018 